\title{
Cefazolin bolus and continuous administration for elective cardiac surgery: Improved pharmacokinetic and pharmacodynamic parameters
}

\author{
C. Adembri, MD, PhD, ${ }^{a}$ R. Ristori, MD, ${ }^{b}$ C. Chelazzi, MD, ${ }^{a}$ S. Arrigucci, BSc, ${ }^{c}$ M. I. Cassetta, BSc, ${ }^{c}$ \\ A. R. De Gaudio, MD, and A. Novelli, MD ${ }^{\mathrm{c}}$
}

\begin{abstract}
Objective: Cefazolin (1-2 g bolus at induction possibly repeated after cardiopulmonary bypass) remains the standard for antibiotic prophylaxis in cardiac surgery. Data indicate, however, that it is underdosed with this dosing schedule. A prospective, randomized study comparing intermittent versus loading dose plus continuous infusion for the same total dose of cefazolin was performed to assess which modality is pharmacokinetically and pharmacodynamically advantageous.
\end{abstract}

\begin{abstract}
Methods: Patients received $2 \mathrm{~g}$ cefazolin as a starting dose and then were divided into an intermittent group (receiving another $1 \mathrm{~g}$ at 3,9 , and 15 hours after the first dose) and a continuous group (continuous infusion started after the first dose, providing $1 \mathrm{~g}$ every 6 hours for 18 hours). Cefazolin levels were measured in blood and atria.

Results: Mean total and calculated free trough concentrations in blood varied greatly among patients in the intermittent group and were lower than those in the continuous group $(P<.05$ at 15,18 and 24 hours). For 9 of 10 $(90 \%)$ patients in the continuous infusion group, the targeted pharmacokinetic and pharmacodynamic goal (time above minimal inhibitory concentration $>90 \%$ ) was achieved, whereas the goal was met for only 3 of $10(30 \%)$ in the intermittent group $(P<.05)$. The mean atrial tissue concentration was also higher with continuous infusion $(P<.05)$.
\end{abstract}

Conclusions: Administration of cefazolin as bolus plus continuous infusion has pharmacokinetic and pharmacodynamic advantages relative to intermittent administration. It provides more stable serum levels, lower interpatient variability, and higher myocardial tissue penetration. (J Thorac Cardiovasc Surg 2010;140:471-5)

Surgical site infections (SSIs) complicate elective cardiac surgery in $3 \%$ to $10 \%$ of cases. ${ }^{1-4}$ Cefazolin, because of its spectrum of activity, safety, and low cost, is still the standard drug of choice for antibiotic prophylaxis in elective cardiac surgery, unless the patient is allergic to $\beta$-lactams or there is a high (usually $>20 \%$ ) prevalence of methicillin-resistant Staphylococcus aureus infection..$^{5}$ It is well known that the success of antibiotic prophylaxis depends not only on selection of the appropriate antimicrobial drug but also on its administration so that peak blood concentrations are achieved before skin incision and adequate levels are maintained during the whole surgical procedure and early postoperative period. ${ }^{5}$ Time-dependent antibacterial agents such as cefazolin should reach and maintain concentrations above the minimal inhibitory concentration

\footnotetext{
From the Section of Anesthesiology and Intensive Care, ${ }^{\mathrm{a}}$ Department of Critical Care, University of Florence, Firenze, Italy; and Casa di Cura Villa Maria Beatrice Hospital, ${ }^{\mathrm{b}}$ Firenze, Italy; and Department of Preclinical and Clinical Pharmacology, ${ }^{\mathrm{c}}$ Firenze, Italy.

Supported by Ministero della Ricerca grant 20078RZT99_004 to C.A. and A.N. Disclosures: None.

Received for publication Aug 17, 2009; revisions received Jan 18, 2010; accepted for publication March 9, 2010; available ahead of print June 7, 2010.

Address for reprints: C. Adembri, MD, PhD, University, Critical care, viale Morgangni

85, 50134 Florence, Italy (E-mail: chiara.adembri@unifi.it).

$0022-5223 / \$ 36.00$

Copyright (C) 2010 by The American Association for Thoracic Surgery

doi:10.1016/j.jtcvs.2010.03.038
}

(MIC) for at least $40 \%$ to $50 \%{ }^{6}$-or even better, for more than $90 \%{ }^{6,7}$-of the dosing interval. In cardiac surgery, cefazolin is usually administered intermittently as a $1-$ to 2-g intravenous bolus before induction of anesthesia, possibly repeated after onset of cardiopulmonary bypass (CPB) or at wound closure. ${ }^{5}$ Drug plasma levels may be profoundly altered during $\mathrm{CPB}$, however, mainly as a result of increased volume of distribution, resulting in underdosing. ${ }^{8}$ As shown by Caffarelli and colleagues, ${ }^{9} 1 \mathrm{~g}$ cefazolin administered at induction and repeated at wound closure provides plasma levels lower than $8 \mu \mathrm{g} / \mathrm{mL}$ (sensitivity threshold for cefazolin ${ }^{10}$ ) in $50 \%$ of patients undergoing CPB for longer than 120 minutes. Similarly, in patients undergoing coronary artery bypass grafting, plasma levels of cefazolin after administration of $1 \mathrm{~g}$ at induction and $1 \mathrm{~g}$ immediately after onset of CPB fluctuated considerably during the intraoperative period, falling below the $90 \%$ MIC for Enterobacter species and Escherichia coli in most patients. ${ }^{1}$ These data indicate that the drug is frequently underdosed, with the risk of both failure to prevent SSIs and a possible increase in the spread of resistance. ${ }^{11}$ To compensate for cefazolin's short elimination half-life, ${ }^{12}$ we hypothesized that it could be administered as a loading bolus followed by continuous infusion. This study was therefore performed to assess which administration schedule (intermittent vs loading dose plus continuous infusion) of the same amount of 


\section{Abbreviations and Acronyms \\ $\mathrm{CPB}=$ cardiopulmonary bypass \\ MIC $=$ minimal inhibitory concentration \\ SSI $=$ surgical site infections}

cefazolin would yield a better pharmacokinetic and pharmacodynamic profile as antibiotic prophylaxis in patients undergoing cardiac surgery with CPB.

\section{MATERIALS AND METHODS}

This prospective, randomized study was performed at a universityaffiliated hospital. The study was approved by the hospital ethics committee, and patients gave informed consent to participate in the study. Enrolled patients were divided into groups according to the modality of cefazolin administration, intermittent or continuous. Exclusion criteria were as follows: age younger than 18 years, pregnancy, suspected or confirmed intolerance to cephalosporins, severe renal failure (creatinine clearance $<40 \mathrm{~mL} / \mathrm{min}$, calculated according to Cockcroft and Gault formula ${ }^{13}$ ), liver failure (serum bilirubin concentration $>2 \mathrm{mg} / \mathrm{dL}$ ), neutropenia (neutrophil count $<500$ cells $/ \mathrm{mm}^{3}$ ), and diabetes (a specific risk factor for development of SSIs, independent of type of surgery or schedule of prophylaxis administration). Patients with preexisting infections or history of antibiotic therapy during the last 72 hours before surgery were excluded as well.

Patients in both groups were preoperatively scored according to the EuroSCORE ${ }^{14}$ to predict operative mortality. All received the same type of anesthesia and intraoperative management. In particular, fluid balance was strictly monitored, with crystalloids and colloids used as fluid replacement in a 3:1 ratio. After surgery, all patients were admitted to the cardiothoracic surgical intensive care unit, where they received the same postoperative care. Fluids were infused to maintain a urinary output of at least $1.5 \mathrm{~mL} /(\mathrm{kg} \cdot \mathrm{h})$. The type of surgery, duration of surgery, and duration of mechanical ventilation were recorded. The presence of SSIs was monitored for 30 days after surgery. Bacteriologic examinations were performed when SSI was suspected.

Patients in both the intermittent and continuous infusion groups received (through a dedicated peripheral venous line) a $2-\mathrm{g}$ dose of cefazolin as a starting dose 30 minutes before skin incision. In the intermittent group, additional 1-g doses of cefazolin were administered at the end of CPB (second dose) and at 9 and 15 hours after the second dose. In the continuous infusion group, a continuous infusion of $3 \mathrm{~g}$ cefazolin was started immediately after the loading dose at a rate of $1 \mathrm{~g}$ every 6 hours (Figure 1). Patients in both groups received a total of $5 \mathrm{~g}$ of cefazolin as prophylaxis.

To assay cefazolin serum levels, blood samples $(3 \mathrm{~mL})$ were collected through an intra-arterial catheter placed contralaterally to the infusion site. In both groups, blood samples were taken just before the loading dose infusion, after completion of loading dose ( 0.5 hours, peak concentration), and then at each trough and peak time for the intermittent group or at the corresponding times for the continuous infusion group for 24 hours (Figure 1). The samples were centrifuged and frozen at $-80^{\circ} \mathrm{C}$. To determine cefazolin myocardial tissue penetration, samples of right atrial auricula were also taken from some patients at the end of CPB. Cefazolin concentrations in serum and myocardial tissue were determined in triplicate by a validated large-plate agar diffusion technique, with a lower limit of sensitivity of $0.125 \mathrm{mg} / \mathrm{L}$, ${ }^{15}$ according to Good Laboratory Practice standards. The correlation coefficient was not less than 0.99 . For all cefazolin samples, intra-assay precision ranged from $1.5 \%$ to $6.8 \%$. Pharmacokinetic analysis of cefazolin concentrations in serum was performed with a computerized program (Syphar, version 4.0; SIMED, Creteil-Cedex, France). The time when free serum concentrations were greater than the MIC (considering $80 \%$ protein binding ${ }^{12}$ ) and the areas under the concentration curve relative to MIC were calculated for each patient for the pharmacokinetic and pharmacodynamic analysis.

Data are reported as mean $\pm S D$ if not otherwise indicated. Demographic data were analyzed with 2-tailed Student $t$ test. One-way analysis of variance and Duncan post hoc test were used for pharmacokinetic analysis.

\section{RESULTS}

Ten patients in each group were enrolled in and completed the study, and there were no differences between groups in demographic characteristics (Table 1). No patients had major intraoperative complications or adverse reactions to cefazolin. No patients underwent renal replacement therapy. At 30 days after surgery, no patients had acquired a SSI.

The serum concentration time courses of individual patients in the intermittent and continuous infusion groups are depicted in Figure 2 ( $A$ and $B$, respectively). Trough serum concentrations are reported in Table 2 . Peak concentrations after the starting dose were similar $(184.2 \pm 19.8 \mathrm{mg} / \mathrm{L}$ vs $165.4 \pm 29.9 \mathrm{mg} / \mathrm{L}$ for intermittent and continuous infusion groups, respectively, difference not significant), as were the areas under the curve for 0 to 24 hours $(1550.9 \pm 310.2$ vs $1699.7 \pm 204.5$, difference not significant). In the intermittent group, there was wide intragroup fluctuation in total trough cefazolin concentration, with values in individual patients as low as $5.2 \mathrm{mg} / \mathrm{L}$ at 14.5 hours, $8.7 \mathrm{mg} / \mathrm{L}$ at 21 hours, and $0 \mathrm{mg} / \mathrm{L}$ at 24 hours (Figure 2 and Table 2). In contrast, the average intrapatient total concentration was stable (between $51.3 \pm 2 \mathrm{mg} / \mathrm{L}$ and $57.6 \pm 13 \mathrm{mg} / \mathrm{L}$ ) during the entire 24-hour study period in the continuous infusion group, with the lowest value being $27.5 \mathrm{mg} / \mathrm{L}$. The differences in serum levels reflect the significant difference in mean total cefazolin concentrations in myocardial tissue between the intermittent and continuous infusion groups $(3.28 \pm 0.1 \mathrm{mg} / \mathrm{L}$ in the intermittent group vs $6.9 \pm 1.1 \mathrm{mg} / \mathrm{L}$ in the continuous infusion group, $\mathrm{n}=6$ each group, $P<.05)$. Looking at the calculated (considering $80 \%$ protein binding ${ }^{12}$ ) concentrations of the free drug, all mean trough values were lower than $8 \mathrm{mg} / \mathrm{L}$ in the intermittent group, and corresponding values were always greater than $10 \mathrm{mg} / \mathrm{L}$ in the continuous infusion group. Fifty percent of patients in the intermittent group had serum levels lower than $8 \mathrm{mg} / \mathrm{L}$ (the susceptibility breakpoint for $E$ coli) for the entire study period, and $25 \%$ had levels lower than $4 \mathrm{mg} / \mathrm{L}$ (the susceptibility breakpoint for $S$ aureus). The pharmacokinetic and pharmacodynamic analysis of free concentrations (Figure 3) showed that in the intermittent group only 3 of 10 patients $(30 \%)$ had concentrations above the MIC threshold of $8 \mathrm{mg} / \mathrm{L}$ more than $90 \%$ of the time. In contrast, $90 \%$ of patients in the continuous infusion group (9/10) had values greater than the MIC more than $90 \%$ of the time $(P<.01)$.

\section{DISCUSSION}

We found that a better pharmacokinetic and pharmacodynamic profile emerged from administering cefazolin as 


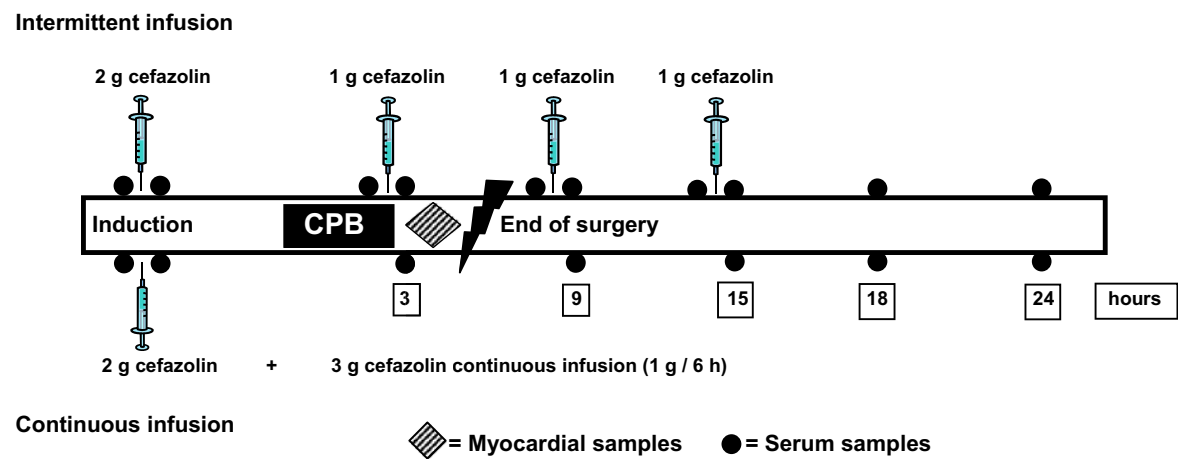

FIGURE 1. Experimental design time course. $C P B$, Cardiopulmonary bypass.

a bolus followed by continuous infusion rather than according to the standard administration schedule as prophylaxis in elective cardiac surgery. Continuous infusion provided serum levels more consistently above the target (greater than MIC for $>90 \%$ of the time) for the most likely susceptible pathogens in all patients, and the duration of effective cefazolin serum level was extended without any increase in the total amount of drug given. Finally, myocardial tissue penetration of the drug was enhanced with continuous administration relative to intermittent administration.

Guidelines for thoracic and cardiovascular surgery still recommend cefazolin as first-line prophylaxis, to be administered intermittently as boluses of 1 to $2 \mathrm{~g}$ at induction and possibly repeated at the end of $\mathrm{CPB}$ or at wound closure. ${ }^{5} \mathrm{Be}-$ cause of its short half-life ( $<2$ hours) and because of pharmacokinetic alterations caused by CPB that result in $30 \%$ to $40 \%$ decreases in plasma levels of hydrophilic drugs, standard dosages at fixed intervals are often suboptimal, even in the intraoperative period, and expose the patient to a higher

TABLE 1. Clinical and demographic characteristics of patients receiving continuous or intermittent cefazolin infusion

\begin{tabular}{lcc}
\hline & Intermittent & Continuous \\
\hline Sex (male/female) & $8: 2$ & $7: 3$ \\
Age $(\mathrm{y}$, mean $\pm \mathrm{SD})$ & $67.9 \pm 9.6$ & $61.9 \pm 9.5$ \\
Body mass index $\left(\mathrm{kg} / \mathrm{m}^{2}\right.$, & $27.4 \pm 3$ & $25.5 \pm 3$ \\
$\quad$ mean $\pm \mathrm{SD}$ ) & & \\
$\quad$ Total proteins $(\mathrm{g} / \mathrm{dL}$, mean $\pm \mathrm{SD})$ & $7.37 \pm 0.5$ & $7.3 \pm 0.3$ \\
$\quad$ Creatinine clearance (mL/min, & $67 \pm 12$ & $75 \pm 16$ \\
$\quad$ mean $\pm \mathrm{SD}$ ) & & \\
EuroSCORE & 3.5 & \\
$\quad$ Median & $2-6$ & 3.0 \\
$\quad$ Range & & \\
Type of surgery (no.) & 5 & 5 \\
$\quad$ CABG & 3 & 2 \\
$\quad$ Valve replacement & 2 & 3 \\
$\quad$ CABG and valve replacement & $4.3 \pm 0.6$ & $4.7 \pm 0.7$ \\
Duration of surgery (h, mean $\pm \mathrm{SD})$ & & \\
Duration of cardiopulmonary bypass & $48.6 \pm 9.2$ & $47.2 \pm 13$ \\
$\quad$ (min, mean \pm SD) & & \\
\hline All differences are nonsignificant. CABG, Coronary artery bypass grafting.
\end{tabular}

risk of sternal SSIs when only $1 \mathrm{~g}$ is administered. ${ }^{16}$ Neither $1 \mathrm{~g}$ cefazolin given before surgery nor a second dose of $1 \mathrm{~g}$ just after initiation of CPB can guarantee optimum cefazolin serum levels for prophylaxis. ${ }^{17}$ Although cefazolin is a timedependent molecule, this problem might be overcome by administering higher doses, since faster bacterial killing is observed when plasma levels are high because the drug can bind simultaneously to multiple penicillin-binding proteins. ${ }^{18}$ Intermittent high doses, such as $4 \mathrm{~g}$ before skin incision and $2 \mathrm{~g}$ at skin closure, although ensuring higher mean peak plasma total concentrations, do not, however, prevent significant interindividual variability, with trough levels below the optimal pharmacokinetic and pharmacodynamic target in the interstitial fluid. ${ }^{19}$ The mean total concentrations varied little and were always greater than $50 \mathrm{mg} / \mathrm{L}$ in our continuous infusion group. Our data agree with those of Waltrip and coworkers, ${ }^{20}$ who studied wound concentrations of cefazolin and found that continuous infusion guaranteed higher serum levels, although our data were obtained with the same total dose of drug in both groups. Moreover, Waltrip and coworkers ${ }^{20}$ did not assay drug concentrations in myocardial tissue, whereas in our study we showed that maintenance of high and stable serum levels was confirmed when higher tissue concentrations were compared.

The duration of prophylaxis in cardiac surgery is controversial. Short-term prophylaxis for clean surgery should cover bacteria at the time of skin incision while minimizing antibiotic use to reduce costs and resistance. On the other hand, longer-term prophylaxis can guarantee antimicrobial coverage during the procedure and in the immediate aftermath, when there is risk of replication of contaminating pathogens. ${ }^{21}$ Nonetheless, it has been found that prolonging prophylaxis for longer than 48 hours does not reduce the incidence of SSIs in cardiac surgery but does increase the spread of resistance. ${ }^{22} \mathrm{We}$ found that continuous infusion provided higher plasma levels than intermittent administration for the first 24 hours without increasing the total amount of drug given.

This study has some limitations. (1) The small number of patients did not provide the power to assess differences in 

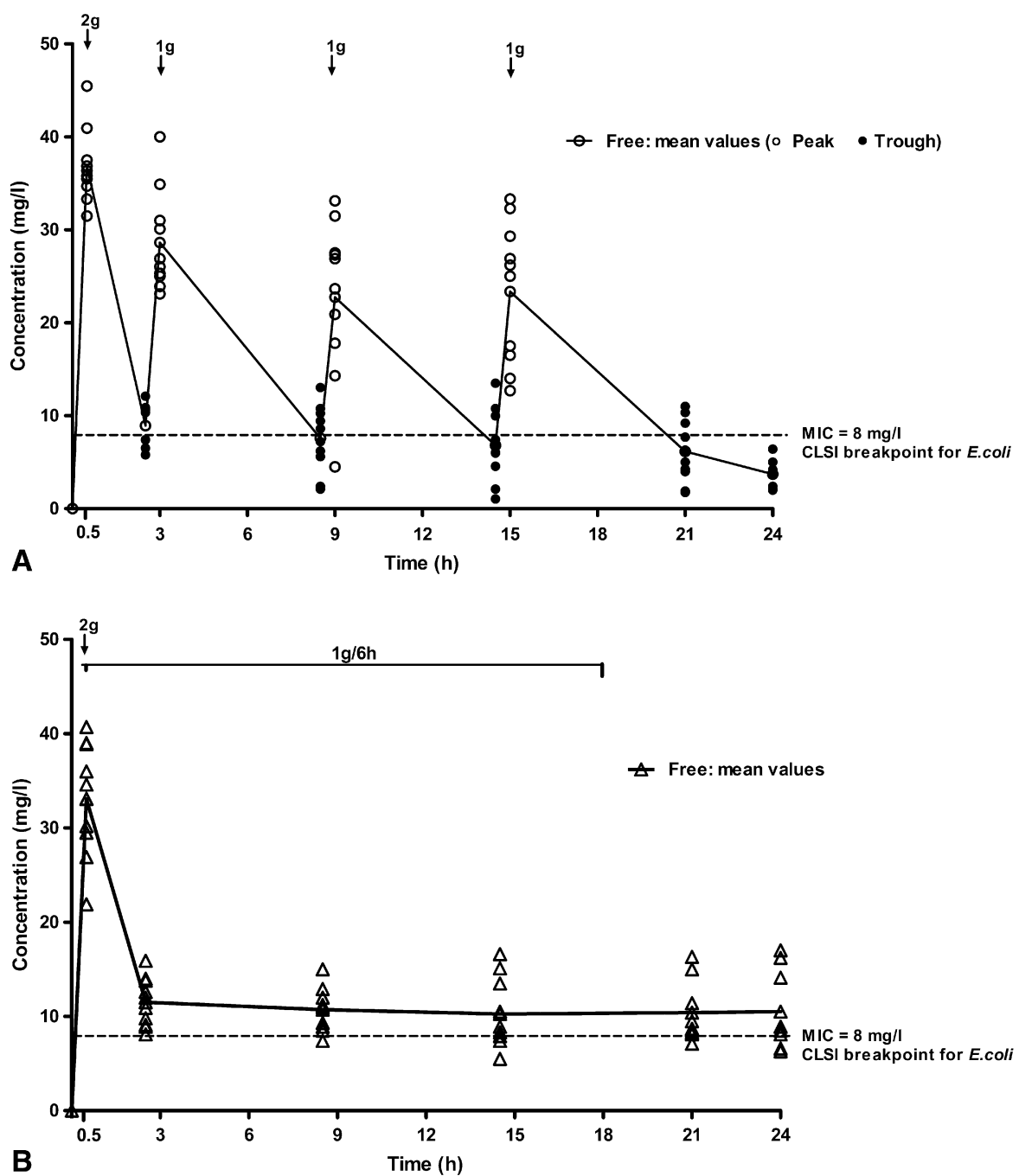

FIGURE 2. Free concentrations of cefazolin in serum. A, Intermittent group. B, Continuous infusion group. MIC, Minimal inhibitory concentration; $C L S I$, Clinical Laboratory Standards Institute.

TABLE 2. Total and free trough serum cefazolin concentrations after intermittent or continuous administration of $5 \mathrm{~g}$ cefazolin

\begin{tabular}{|c|c|c|c|c|}
\hline \multirow[b]{2}{*}{ Time (h) } & \multicolumn{2}{|c|}{ Total concentration } & \multicolumn{2}{|c|}{ Calculated mean free concentration } \\
\hline & Intermittent & Continuous & Intermittent & Continuous \\
\hline \multicolumn{5}{|l|}{2.5} \\
\hline Mean $\pm \mathrm{SD}$ & $44.6 \pm 11.1$ & $57.5 \pm 12.9$ & $8.9 \pm 2.3$ & $11.5 \pm 2.6$ \\
\hline Range & $29-60.5$ & $40.5-79.5$ & $5.8-12.1$ & $8.1-15.9$ \\
\hline \multicolumn{5}{|l|}{8.5} \\
\hline Mean \pm SD & $37.80 \pm 17.8$ & $53.6 \pm 11.3$ & $7.56 \pm 3.56$ & $10.72 \pm 2.27$ \\
\hline Range & $10.5-65.15$ & $37-75$ & $2.1-13.03$ & $7.4-15$ \\
\hline \multicolumn{5}{|l|}{14.5} \\
\hline Mean \pm SD & $34.1 \pm 19.2$ & $51.3 \pm 18.1^{*}$ & $6.8 \pm 3.8$ & $10.3 \pm 3.6^{*}$ \\
\hline Range & $5.2-67.5$ & $27.5-83$ & $1.04-13.5$ & $5.5-16.6$ \\
\hline \multicolumn{5}{|l|}{24} \\
\hline Mean \pm SD & $14.9 \pm 10.3$ & $52.5 \pm 19.4 \dagger$ & $3.7 \pm 1.5$ & $10.51 \pm 3.9 \dagger$ \\
\hline Range & $0-32$ & $31.5-85$ & $0-6.4$ & $6.3-17$ \\
\hline
\end{tabular}




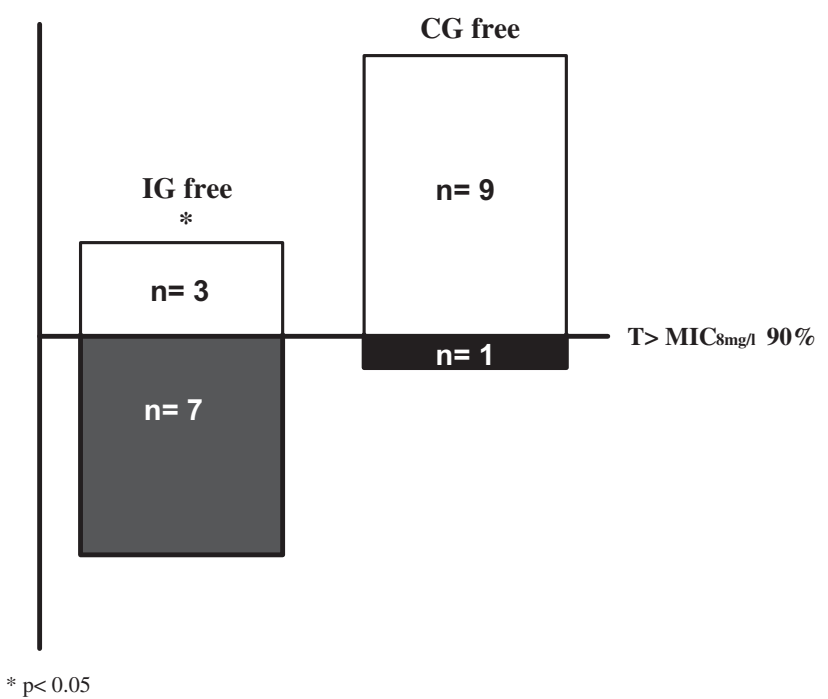

FIGURE 3. Numbers of patients meeting pharmacokinetic and pharmacodynamic target (time $>90 \%$ of dosing interval when free concentration was greater than minimal inhibitory concentration of $8 \mathrm{mg} / \mathrm{L}, T>M I C_{8 m g} / l$ $90 \%$ ) in intermittent group $(I G)$ and continuous infusion group $(C G)$. Asterisk indicates $P<.05$.

the occurrence of SSIs, even though the groups were well matched, surgery was performed by the same skilled operator, and surgical time was standardized. On the other hand, this was mainly a pharmacokinetic and pharmacodynamic study, and the differences in pharmacokinetics and pharmacodynamics that emerged between the administration modalities are noteworthy. (2) We gave the same dose of cefazolin regardless of the patient's body weight. Patients' mean body mass index values were, however, similar between groups. (3) Cefazolin serum levels were measured with a microbiologic method, which is less specific than high-performance liquid chromatography. Intragroup variability, however, was low $(1.5 \%-6.8 \%)$. (4) The selection of $8 \mathrm{mg} / \mathrm{L}$ as the target threshold concentration of free serum cefazolin was somewhat arbitrary. It does, however, correspond to the $90 \%$ MIC for $S$ aureus and the MIC for $E$ coli.

Despite these limitations, our data confirm that giving cefazolin as a bolus followed by continuous infusion in cardiac patients undergoing CPB achieves targeted pharmacokinetic and pharmacodynamic parameters and better myocardial tissue penetration in more patients without increased cost or higher total dose. Although this study did not show a reduced number of SSIs, optimization the pharmacokinetic and pharmacodynamic profile of an "old" but still useful drug, without any additional cost, does appear to be an appealing strategy to preserve its efficacy.
We thank Mary Forrest for editing the manuscript.

\section{References}

1. Fernandez-Ayala M, Nan DN, Farinas-Alvarez C, Revuelta JM, GonzalezMacias J, Farinas MC. Surgical site infection during hospitalization and after discharge in patients who have undergone cardiac surgery. Infect Control Hosp Epidemiol. 2006;27:85-8.

2. Harrington G, Russo P, Spelman D, Borrell S, Watson K, Barr W, et al. Surgicalsite infection rates and risk factor analysis in coronary artery bypass graft surgery. Infect Control Hosp Epidemiol. 2004;25:472-6.

3. Lepelletier D, Perron S, Bizouarn P, Caillon J, Drugeon H, Michaud JL, et al. Surgical-site infection after cardiac surgery: incidence, microbiology, and risk factors. Infect Control Hosp Epidemiol. 2005;26:466-72.

4. Ridderstolpe L, Gill H, Granfeldt H, Ahlfeldt H, Rutberg H. Superficial and deep sternal wound complications: incidence, risk factors and mortality. Eur J Cardiothorac Surg. 2001;20:1168-75.

5. Engelman R, Shahian D, Shemin R, Guy TS, Bratzler D, Edwards F, et al. The Society of Thoracic Surgeons practice guideline series: Antibiotic prophylaxis in cardiac surgery, part II: Antibiotic choice. Ann Thorac Surg. 2007;83:1569-76.

6. Schaper KJ, Schubert S, Dalhoff A. Kinetics and quantification of antibacterial effects of beta lactams, macrolides, and quinolones against gram-positive and gram-negative RTI pathogens. Infection. 2005;33(Suppl. 2):3-14.

7. Nicolau DP, Quintiliani R. Choosing between the new cephalosporin antibiotics: a pharmacodynamic approach. Pharmacoeconomics. 1994;5(Suppl 2):34-9.

8. Buylaert WA, Herregods LL, Mortier EP, Bogaert MG. Cardiopulmonary bypass and the pharmacokinetics of drugs. An update. Clin Pharmacokinet. 1989;17: 10-26.

9. Caffarelli AD, Holden JP, Baron EJ, Lemmens HJ, D'Souza H, Yau V, et al. Plasma cefazolin levels during cardiovascular surgery: effects of cardiopulmonary bypass and profound hypothermic circulatory arrest. $J$ Thorac Cardiovasc Surg. 2006;131:1338-43.

10. Clinical Laboratory Standards Institute (CLSI), National Committee for Clinical Laboratory Standards. Performance standards for antimicrobial susceptibility testing; fourteenth international supplement M100-S14. Wayne (PA): The Committee; 2004.

11. Adembri C, Novelli A. Pharmacokinetic and pharmacodynamic parameters of antimicrobials: potential for providing dosing regimens that are less vulnerable to resistance. Clin Pharmacokinet. 2009;48:517-28.

12. Moellering RC Jr, Swartz MN. Drug therapy: the newer cephalosporins. $N$ Engl J Med. 1976;294:24-8.

13. Cockcroft DW, Gault MH. Prediction of creatinine clearance from serum creatinine. Nephron. 1976;16:31-41.

14. Nashef SA, Roques F, Hammill BG, Peterson ED, Michel P, Grover FL, et al. Validation of European System for Cardiac Operative Risk Evaluation (EuroSCORE) in North American cardiac surgery. Eur J Cardiothorac Surg. 2002;22:101-5.

15. Mazzei T, Tonelli F, Novelli A, Ficari F, Mazzoni C, Anastasi A, et al. Penetration of cefotetan into suction skin blister fluid and tissue homogenates in patients undergoing abdominal surgery. Antimicrob Agents Chemother. 1994;38:2221-3.

16. Lehot JJ. Cefazolin prophylaxis during cardiac operations. Ann Thorac Surg. 2004;77:755-6.

17. Fellinger EK, Leavitt BJ, Hebert JC. Serum levels of prophylactic cefazolin during cardiopulmonary bypass surgery. Ann Thorac Surg. 2002;74:1187-90.

18. Periti P, Nicoletti P. Classification of betalactam antibiotics according to their pharmacodynamics. J Chemother. 1999;11:323-30.

19. Hutschala D, Skhirtladze K, Kinstner C, Mayer-Helm B, Müller M, Wolner E, et al. In vivo microdialysis to measure antibiotic penetration into soft tissue during cardiac surgery. Ann Thorac Surg. 2007;84:1605-10.

20. Waltrip T, Lewis R, Young V, Farmer M, Clayton S, Myers S, et al. A pilot study to determine the feasibility of continuous cefazolin infusion. Surg Infect. 2002;3 5-9.

21. Novelli A. Antimicrobial prophylaxis in surgery: the role of pharmacokinetics. J Chemother. 1999;11:565-72.

22. Harbarth S, Samore MH, Lichtenberg D, Carmeli Y. Prolonged antibiotic prophylaxis after cardiovascular surgery and its effect on surgical site infections and antimicrobial resistance. Circulation. 2000;27:2916-21. 